\title{
SLEEP-DEPENDENT MOTOR MEMORY PLASTICITY IN THE HUMAN BRAIN
}

\author{
M. P. WALKER, ${ }^{a, b *}$ R. STICKGOLD, ${ }^{b}$ D. ALSOP, ${ }^{c}$ \\ N. GAAB $^{\text {d }}$ AND G. SCHLAUG \\ aSleep and Neuroimaging Laboratory, FD/Feldberg 862, Department \\ of Psychiatry, Beth Israel Deaconess Medical Center, Harvard Medical \\ School, Boston, MA 02115, USA \\ ${ }^{b}$ Center for Sleep and Cognition, Department of Psychiatry, Beth Israel \\ Deaconess Medical Center, Harvard Medical School, Boston, MA \\ 02115, USA \\ ${ }^{c}$ Department of Radiology, Beth Israel Deaconess Medical Center, \\ Harvard Medical School, Boston, MA 02115, USA \\ ${ }^{d}$ Department of Neurology, Beth Israel Deaconess Medical Center, \\ Harvard Medical School, Boston, MA 02115, USA
}

Abstract-Growing evidence indicates a role for sleep in off-line memory processing, specifically in post-training consolidation. In humans, sleep has been shown to trigger overnight learning on a motor-sequence memory task, while equivalent waking periods produce no such improvement. But while the behavioral characteristics of sleep-dependent motor learning become increasingly well characterized, the underlying neural basis remains unknown. Here we present functional magnetic resonance imaging data demonstrating a change in the representation of a motor memory after a night of sleep. Subjects trained on a motor-skill memory and 12 hours later, after either sleep or wake, were retested during functional magnetic resonance imaging. Following sleep relative to wake, regions of increased activation were expressed in the right primary motor cortex, medial prefrontal lobe, hippocampus and left cerebellum; changes that can support faster motor output and more precise mapping of key-press movements. In contrast, signal decreases were identified in parietal cortices, the left insular cortex, temporal pole and fronto-polar region, reflecting a reduced need for conscious spatial monitoring and a decreased emotional task burden. This evidence of an overnight, systemslevel change in the representation of a motor memory holds important implications for acquiring real-life skills and in clinical rehabilitation following brain trauma, such as stroke. (c) 2005 IBRO. Published by Elsevier Ltd. All rights reserved.

Key words: sleep, plasticity, motor learning, memory representation, memory reorganization.

A large body of evidence, spanning a wide range of neuroscientific disciplines, now describes evidence of sleepdependent learning in both humans and animals (Walker and Stickgold, 2004), already complemented by cellular

${ }^{*}$ Correspondence to: M. P. Walker, Sleep and Neuroimaging Laboratory, FD/Feldberg 862, Department of Psychiatry, Beth Israel Deaconess Medical Center, Harvard Medical School, Boston, MA 02115, USA. Tel: +1617-667-8486; fax: +1-617-667-8498

E-mail address: mwalker@hms.harvard.edu (M. P. Walker).

Abbreviations: fMRI, functional magnetic resonance imaging; $\mathrm{M} 1$, primary motor cortex; NREM, non-rapid eye movement sleep; SPM99, statistical parametric mapping 99. and molecular models of sleep-dependent plasticity (Graves et al., 2001; Tononi and Cirelli, 2001; Benington and Frank, 2003). In particular, sleep has been implicated in the ongoing process of consolidation, following initial memory acquisition.

Within the procedural memory domain, sleep in humans has been shown to trigger significant overnight learning enhancements, whereby performance is selectively improved across sleeping intervals, while equivalent waking periods confer no such performance benefit (for reviews see Walker, in press). Demonstrations of overnight, sleep-dependent learning have now been reported across both sensory (Karni et al., 1994; Gais et al., 2000; Stickgold et al., 2000a,b; Fenn et al., 2003; Atienza et al., 2004; Gaab et al., 2004) and motor (Smith and MacNeill, 1994; Fischer et al., 2002; Walker et al., 2002, 2003a,b; Korman et al., 2003; Huber et al., 2004; Robertson et al., 2004; Kuriyama et al., 2004) skill memory domains.

Regarding motor-sequence learning, Walker et al. (2002, $2003 a, b)$ have shown that a night of sleep can trigger significant improvements in both performance speed and accuracy on a finger-tapping task, while equivalent periods of time awake do not result in any such learning enhancements. Furthermore, these overnight learning gains correlated with the amount of stage two non-rapid eye movement (NREM) sleep, particularly late in the night (Walker et al., 2002). Adding to these findings, it also appears that there is no transfer of sleep-dependent procedural learning to either new motor sequences, or to performance of the same sequence using the opposite hand (Fischer et al., 2002; Korman et al., 2003), suggesting that the influence of sleep is highly specific. But while the behavioral characteristics of sleep-dependent motor learning are increasingly well characterized, the underlying neural basis of these overnight improvements remains unknown.

Several studies have examined long-term changes in brain activity associated with motor learning, independent of sleep, but following continued practice across days/weeks. These reports have described increased activity or responsiveness in the primary motor cortex (M1), and to a lesser extent, the pre-motor cortex, basal ganglia and cerebellum (Karni et al., 1995; Pascual-Leone et al., 1995; Penhune and Doyon, 2002). Enhanced motor skill learning has also been associated with signal decreases throughout the parietal cortex, thought to reflect automaticity of performance as skill level improves (Seitz et al., 1990; Toni et al., 1998; Muller et al., 2002; Sakai et al., 2002). Despite these findings, however, no study has yet explored the beneficial effect of a night of sleep on delayed motor-sequence learning and associated plastic brain changes, or examined alterations in brain plas- 
ticity following episodes of sleep relative to equivalent waking periods in an attempt to understanding the neural basis of sleep-dependent learning.

Using functional magnetic resonance imaging (fMRI), here we investigate the hypothesis that following initial memory acquisition, overnight sleep triggers a significant shift or reorganization of the memory representation relative to an equivalent intervening time awake. Since learning of a specific motor-sequence on this task does not transfer to a newly encountered motor-sequence using the same hand (Fischer et al., 2002), we were able to use several different motor-sequences to investigate the contributions of wake and sleep in the same subjects using a counterbalanced, crossover design (see Experimental Procedures for details). Based on previous psychophysical and functional imaging studies of motor skill learning, we hypothesized that intervening sleep would result in significantly greater signal intensity within corresponding motor cortical areas, particularly the $\mathrm{M} 1$, and in the cerebellar cortex. In contrast, it was also hypothesized that there would be corresponding decreased signal intensity within the parietal cortices as a consequence of greater task automaticity, post-sleep (Kuriyama et al., 2004).

\section{EXPERIMENTAL PROCEDURES}

The experiment was approved by the local human studies committee, and in accordance with the principles of the Declaration of Helsinki.

\section{Participants}

Subjects ( $n=12$; seven females, five males, mean age 23.3, S.D. \pm 1.9 ) had no prior history of drug or alcohol abuse, neurological, psychiatric or sleep disorders, and agreed to be drug, alcohol and caffeine free for $24 \mathrm{~h}$ prior to and during the study period. All subjects were right-handed, and maintained a standard sleep schedule for 1 week prior to the study. On the intervening night of sleep during the experimental phase, subjects obtained an average $8.1 \mathrm{~h}$ sleep (S.D. \pm 0.83 ), as measured by sleep-log diaries.

\section{Experimental protocol}

We utilized the same finger-tapping task employed in our prior investigations (Walker et al., 2002, 2003a,b), discussed in Fig. 1.

Subjects entered a repeated measures design, composed of a "NIGHT-SLEEP" phase and a "DAY-WAKE" phase, presented in a counterbalanced crossover design, described in detail in Fig. 1. In brief, during the NIGHT-SLEEP phase, subjects trained on a motor-sequence in the evening, and $12 \mathrm{~h}$ later, following a night of sleep, were retested during fMRI scanning. After retesting, subjects trained and immediately retested on a different motor-sequence during fMRI scanning, serving as an AM circadian control.

In the DAY-WAKE phase, subjects repeated these events, first undergoing an initial training session on a new motor-sequence, but in the morning. Twelve hours later, without intervening sleep, subjects retested on this motor-sequence during fMRI scanning. Immediately following, subjects trained and then retested on a different motor-sequence, which served as a PM circadian control.

These two phases were counterbalanced; so that half of the subjects underwent the DAY-WAKE phase first, followed by the NIGHT-SLEEP phase, while the remaining half experienced the NIGHT-SLEEP phase first, followed by the DAY-WAKE phase, with the two always being separated by a 1 week interval. Thus,

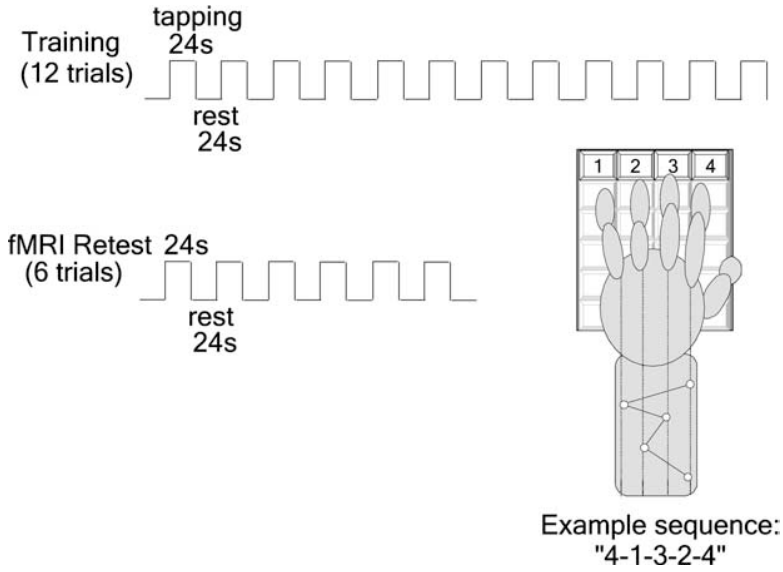

Fig. 1. Motor skill task. The task was composed of a block paradigm containing multiple trials, with each trial consisting of an active "tapping" period (24 s) and a "rest" period (24 s). Tapping periods required subjects to repeatedly type a specific five-element numerical sequence (e.g. 4-1-3-2-4) on a numbered button box with the fingers of the non-dominant (left) hand, with the computer scoring for the number of complete sequences achieved ("speed"), and the number of errors made relative to the number of sequences (errors/sequence; "error rate"). Training sessions (no MRI scanning) were composed of 12 trials of finger-tapping on a specific motor-sequence, while retest sessions during fMRI scanning were composed of six trials. To circumvent the likelihood that unconstrained retesting following sleep would likely be performed at a higher tapping rate, thereby modifying fMRI blood flow characteristics, we controlled this variable by pacing finger-tapping rate using an auditory metronome cue at $3.1 \mathrm{~Hz}$ during the fMRI retest sessions. This paced tapping rate, which was determined by analyzing the behavioral performance of over 120 previous subjects, allowed for a demanding but manageable performance, importantly standardizing skill rate across conditions. Auditory pacing was presented to subjects via MR compatible headphones during both tapping and rest periods, resulting in cancellation of the auditory stimulation in the fMRI contrasts.

we were able to investigate, in the same subjects, after equivalent amounts of training, and after equivalent amounts of intervening time, how brain activity during retesting on a motor-sequence memory differed following a night of sleep, relative to an equivalent time period awake. In addition, we were also able to assess any diurnal effects on these imaging contrasts by comparing brain activity for equivalently trained motor-sequences at each respective time of day (AM and PM; Fig. 2).

\section{MRI scanning and data analysis}

MRI data were acquired with a $3 T$ GE system (GE Medical Systems, WI, USA). Structural anatomical images were acquired using a T1-weighted gradient echo sequence, covering the whole brain volume with $1.5 \mathrm{~mm}$ sagittal slices, no gap (TE/TR $=3.3$ / $8.2 \mathrm{~ms}, \mathrm{FOV}=24 \mathrm{~cm}$, matrix $=256 \times 128)$. Functional MRI images were acquired using a gradient echo-planar $\mathrm{T} 2{ }^{*}$-sequence sensitive to the blood-oxygenation level-dependent (BOLD) contrast. Functional image volumes consisted of 28 oblique-axial slices (thickness $=4 \mathrm{~mm}$, matrix $=64 \times 64, \mathrm{TR} / \mathrm{TE}=4000 / 25 \mathrm{~ms}$ ) covering the whole brain volume.

fMRI data were analyzed using the statistical parametric mapping (SPM99) software package (www.fil.ion.ucl.ac.uk/spm/ spm99.html). Each set of axial images for each subject was realigned to the first image, co-registered with the corresponding T1-weighted data set, spatially normalized to the SPM99 T1-template, and smoothed with an isotropic Gaussian kernel $(8 \mathrm{~mm}$ full-width at half-maximum). Subject and condition effects were estimated using a general linear model (Friston et al., 1995). Global differences in scan 

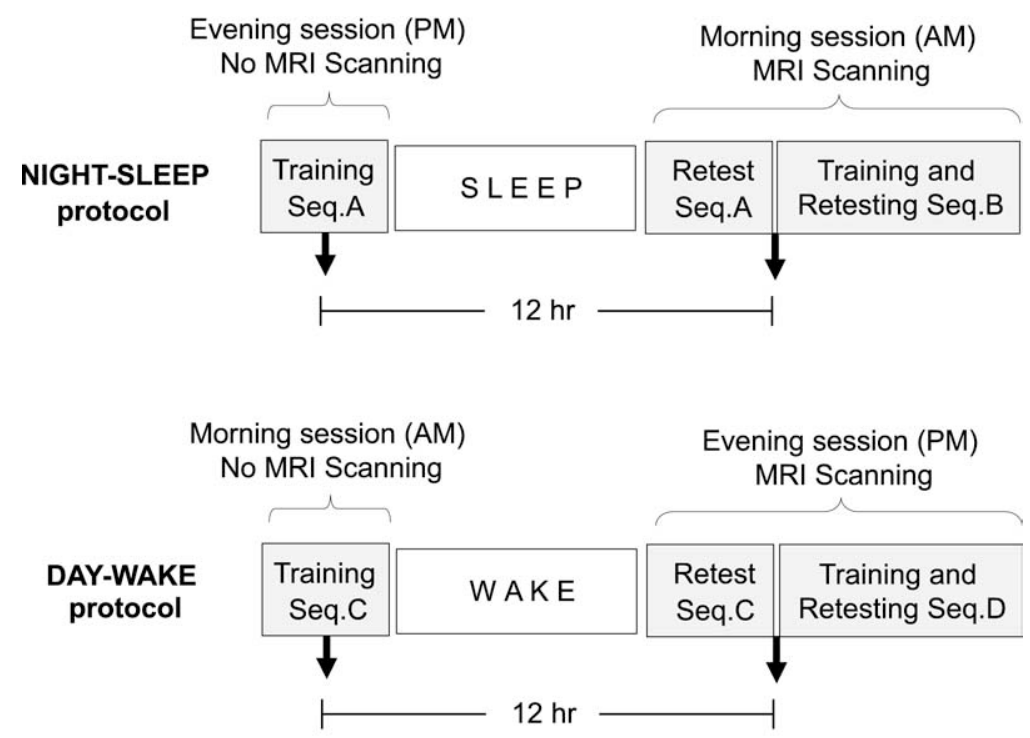

Fig. 2. Experimental protocol. Subjects entered a repeated measures crossover design, composed of a "NIGHT-SLEEP" phase and a "DAY-WAKE" phase, separated by 1 week, and presented in counterbalanced order across subjects. Since learning of a specific motor-sequence does not transfer to a newly encountered motor-sequence using the same hand (Fischer et al., 2002; Walker et al., 2003a), we were able to use several different motor-sequences to investigate the contributions of wake and sleep in the same subjects. Four different five-element motor-sequence patterns (4-1-3-2-4, 2-3-1-4-2, 3-4-2-1-3, 1-4-2-3-1) were used in a counterbalanced order as Seq.A, Seq.B, Seq.C, and Seq.D. These sequences were shown to be equal in difficulty level and learning profile in pilot testing (and see Walker et al., 2003a). In the NIGHT-SLEEP phase, subjects trained on a specific motor-sequence in the evening $(9 \mathrm{PM} \pm 1 \mathrm{hr}$ ) without $\mathrm{fMRI}$ scanning. Twelve hours later, following a night of sleep, subjects performed a retest session on the same motor-sequence during $\mathrm{fMRI}$ scanning. After retesting on the original motor-sequence, subjects trained on a different motor-sequence and were immediately retested during fMRI scanning in the same session. This provided a corresponding, equivalently practiced, AM circadian control condition. In the DAY-WAKE phase, subjects trained on a different motor-sequence in the morning ( $9 A M \pm 1 \mathrm{hr})$, and $12 \mathrm{~h}$ later, without sleep, retested on the same motor-sequence during fMRI scanning. Again, after retesting on this original motor-sequence, subjects trained and were immediately retested on a different motor-sequence during $\mathrm{fMRI}$ scanning. This again offered a corresponding, equivalently practiced, PM circadian control condition. Therefore, activation maps for trained motor-sequence patterns were obtained following sleep and wake, together with activation maps for equivalently trained motor-sequence patterns at the corresponding circadian times (AM and PM).

intensity were removed by scaling each scan in proportion to its global intensity, and low-frequency drifts were removed using the default temporal high-pass filter. Brodmann's area (BA) were identified according to the atlas of Talairach and Tournoux, and cerebellum locations according to Schmahmann et al. (1999). Individual contrast images were produced by comparing task-dependent activation (tapping > rest) in the NIGHT-SLEEP condition and the DAYWAKE condition for each subject separately. Group changes were then determined using a $t$-test performed across these task-dependent images to identify brain areas in which cerebral activity differed between the NIGHT-SLEEP and DAY-WAKE conditions in each direction ([NIGHT-SLEEP $>$ DAY-WAKE] and [NIGHT-SLEEP $<$ DAY-WAKE]), at an applied corrected threshold of $P<0.05^{\mathrm{FWE}}$ to control for multiple comparisons (Worsley et al., 1996). Similarly, for the circadian control comparisons; individual contrast images were firstly produced by comparing task-dependent activation (tapping $>$ rest) in the AM condition and the PM condition for each subject separately. This was followed by group analysis, again determined using a $t$-test across these task-dependent images to identify brain areas in which cerebral activity differed between the AM and PM conditions, also constrained at an applied corrected threshold of $P<0.05^{\mathrm{FWE}}$.

\section{RESULTS}

\section{Behavioral data}

At the end of the initial training sessions in the NIGHT-SLEEP and DAY-WAKE protocols (average of trials 10-12), subjects achieved near identical performance levels (Speed: 17.55

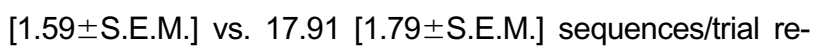
spectively, paired $t$-test $\left[t_{(11)}=0.46, P=0.64\right]$; Error Rate: 0.11 [0.03 \pm S.E.M.] vs. 0.13 [0.02 \pm S.E.M.] errors/sequence respectively, paired $t$-test $\left.\left[t_{(11)}=0.83, P=0.42\right]\right)$. Therefore, the post-training level of acquired skill did not differ between the two conditions, supporting previous reports of a lack of transfer between motor-sequences (Fischer et al., 2002), and the lack of circadian influence on motor performance at these different times (Walker et al., 2002). At the later retest session during $\mathrm{fMRI}$ scanning, finger-tapping was held constant using auditory pacing (see Fig. 2), standardizing tapping rate so as to negating the potential confound of speed differences modify blood flow characteristics. Nevertheless, there was evidence of improved performance accuracy (lower errors/sequence) in the NIGHT-SLEEP condition compared

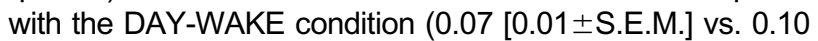
[0.02 \pm S.E.M.]), consistent with previous reports of overnight sleep-dependent consolidation enhancements (Fischer et al., 2002; Korman et al., 2003; Walker et al., 2003a,b, 2002) although this paced task configuration may limit the full expression of such learning.

\section{fMRI group comparisons}

When activation maps from the NIGHT-SLEEP protocol were contrasted with those from the DAY-WAKE protocol (NIGHT-SLEEP>DAY-WAKE), post-sleep regions of in- 

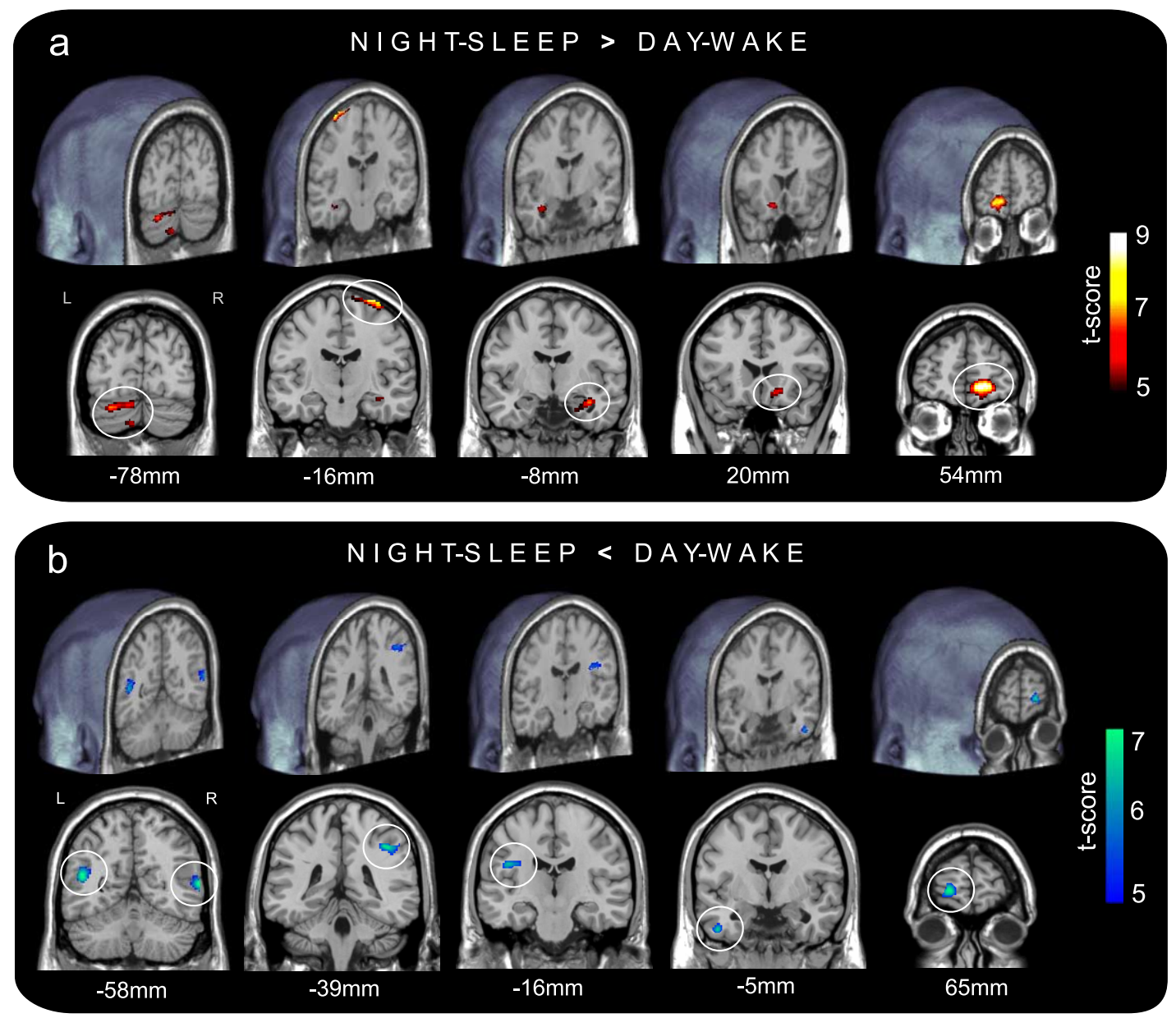

Fig. 3. fMRI group (condition) effects. (A) Increased fMRI signal intensity (in red/yellow) and (B) decreased signal intensity (in blue) during retesting following training and $12 \mathrm{~h}$ containing intervening sleep ("NIGHT-SLEEP"), relative to training followed by $12 \mathrm{~h}$ without intervening sleep ("DAYWAKE"). Differences are displayed on three-dimensional rendered brains (upper graphic of each panel; with mixed orientation views), together with corresponding two-dimensional coronal sections (lower graphic of each panel; using standard L-R convention views, and with regions of activation circled) in ICBM 152 (International Consortium for Brain Mapping) space.

creased activation were observed in the right primary motor cortex (M1) (precentral gyrus), consonant with the fact that subjects were typing with their left, contralateral hand (Fig. 3A).

In addition, there were also regions of increased activity in the right anterior medial prefrontal lobe, right hippocampus, and right ventral striatum, together with regions of the left cerebellar cortex, lobules VI and VII (which control the ipsilateral, left, motor limb) (Fig. 3A).

In the opposite group contrast (NIGHT-SLEEP $<$ DAYWAKE), there were post-sleep regions of decreased signal intensity, bilaterally, in the parietal cortices, largely inferior. Furthermore, there were additional regions of signal decrease in the left insular cortex, left temporal pole and left inferior fronto-polar cortex (Fig. 3B).

Anatomical coordinates for all regions of significant difference, and the corresponding normalized Z-scores, are provided in Table 1.

To exclude the possibility that these changes may also reflect circadian influences on basic performance-related brain activity, we compared task-dependent activation dur- ing retesting of similarly trained motor-sequences at each of these respective circadian time points (AM and PM; see Fig. 2 and Experimental Procedures). That is to say, we established whether any circadian differences in task-dependent brain activity at these times mirrored those changes seen in the NIGHT-SLEEP and DAY-WAKE comparison. This was achieved using an inclusive mask contrast in SPM, specifically designed to determine whether areas showing difference in the $\mathrm{AM}<>\mathrm{PM}$ conditions overlapped with changes expressed in the NIGHTSLEEP $<>$ DAY-WAKE contrasts. In this way, the inclusive mask revealed whether areas of difference were common across the AM $<>$ PM and NIGHT-SLEEP $<>$ DAYWAKE contrasts, or exclusive to one of them. In the AM $>$ PM contrast (the equivalent circadian times as the NIGHTSLEEP $>$ DAY-WAKE contrast), only one single voxel (in M1; coordinates $x=30, y=16, z=76$ ), matched any of the 1366 identified in the NIGHT-SLEEP > DAY-WAKE comparison. In the $\mathrm{AM}<\mathrm{PM}$ contrast (equivalent circadian time of the NIGHT-SLEEP $<$ DAY-WAKE contrast), no matching voxels were found. Thus, almost no regions of overlap in the $\mathrm{AM}<$ 
Table 1. Anatomical coordinates for significant clusters of activation for the main group (condition) comparisons

\begin{tabular}{|c|c|c|c|c|c|}
\hline Region (Brodmann's area) & $x$ & $y$ & $z$ & Cluster size (voxels) & Peak Z-score \\
\hline \multicolumn{6}{|l|}{ NIGHT-SLEEP > DAY-WAKE } \\
\hline M1 - R (BA 4) & 38 & -20 & 73 & 176 & 6.84 \\
\hline \multicolumn{6}{|l|}{ Cerebellum } \\
\hline Lobule VI - posterior cortex - L & -26 & -79 & -36 & 119 & 6.75 \\
\hline Lobule VII - L & -8 & -78 & -52 & 73 & 5.88 \\
\hline Anterior medial prefrontal lobe - R (BA 10) & 16 & 52 & -4 & 650 & 7.57 \\
\hline Hippocampus - R & 32 & -10 & 22 & 99 & 6.05 \\
\hline Ventral striatum - R & 20 & 12 & -13 & 249 & 6.60 \\
\hline \multicolumn{6}{|l|}{ NIGHT-SLEEP < DAY-WAKE } \\
\hline Superior fronto-polar region - L (BA 10/11) & -18 & 68 & 4 & 114 & 7.25 \\
\hline Insular cortex - L (BA 13) & -34 & 14 & 18 & 55 & 5.93 \\
\hline Temporal pole - L (BA 20) & -46 & -2 & -43 & 96 & 6.11 \\
\hline \multicolumn{6}{|l|}{ Parietal cortex } \\
\hline Superior/inferior parietal lobe - R (BA 40) & 40 & -43 & 46 & 106 & 5.91 \\
\hline Inferior parietal lobe - R (BA 39) & 51 & -54 & 12 & 85 & 6.52 \\
\hline Inferior parietal lobe - L (BA 39) & -44 & -56 & 10 & 295 & 6.41 \\
\hline
\end{tabular}

The $x-y-z$ coordinates are given in ICBM ${ }_{152}$ (International Consortium for Brain Mapping) space, together with normalized Z-scores at the peak voxel of intensity. Left or right side designated by $L$ and $R$ respectively. The Brodmann's area (BA) location is identified according to the atlas of Talairach and Tournoux, and cerebellum locations according to Schmahmann et al. (1999).

$>$ PM contrasts were conjointly active in the NIGHTSLEEP $<>$ DAY-WAKE contrasts, suggesting that differences between the NIGHT-SLEEP and DAY-WAKE conditions were not an artifact of diurnal factors influencing performance-related brain activity.

\section{DISCUSSION}

We have identified regionally specific differences in functional activation during retesting on a motor-sequence task following a night of sleep, relative to a corresponding time interval awake. These changes cannot be explained either by 1) differences in initial training, since subjects received the same amount of initial practice, and achieved similar post-training skill levels, or 2) circadian influences on performance-related brain activation, since a separate comparison of task-related activity during retesting on equivalently trained motor-sequences at these respective circadian times revealed less than $0.1 \%$ of overlapping voxels relative to the sleep-wake contrasts. Instead, we believe the most parsimonious explanation of this plastic change to be the specific influence of intervening sleep. Since behavioral studies have shown that post-training sleep, but not wake, leads to enhanced performance on this task (Walker et al., 2002, 2003a,b; Fischer et al., 2002; Korman et al., 2003; Kuriyama et al., 2004), it is suggested that this systems-level plastic change specifically reflects the consequence of sleep-dependent consolidation processes. It should be appreciated, however, that because subjects' performance skill rates were similarly paced in the two conditions during $\mathrm{fMRI}$ retesting, the true differences between sleep and wake conditions during unconstrained motor-skill performance may be even greater than the changes seen here, an important limitation of this study.

The finding of increased activation in M1 following sleep is supportive of our original hypothesis, and extends previous investigations of motor skill learning following continued practice but over much greater time periods (multiple days to weeks not concerning sleep) (Karni et al., 1995; Pascual-Leone et al., 1995; Penhune and Doyon, 2002). Based on these data, it seems reasonable to hypothesize that delayed/off-line motor skill learning is associated with increased functional activity and/or an expansion of the cortical representation in M1 (Ungerleider et al., 2002), and our findings indicate that sleep plays a fundamental role in the evolution of such plastic changes. Similarly, we suggest that the enhanced cerebellar activation reflects a corresponding increased need for error monitoring/prediction, post-sleep, in correspondence with, or response to, the augmented output capabilities of $M 1$ (Ohyama et al., 2003). These overnight changes in the cerebellum, together with the changes in $M 1$, can allow more precise motor output and faster mapping of intention to key-press movements, and offer a cogent neural basis for previously reported sleep-dependent behavioral improvements in both performance speed and accuracy on this task (Walker et al., 2002, 2003a,b; Fischer et al., 2002; Korman et al., 2003; Kuriyama et al., 2004).

Several additional regions of difference were observed that were not included in our original hypotheses. Firstly, increased post-sleep hippocampal activation was observed following a night of sleep; a finding that is of particular interest in light of recent evidence that sequence learning (Poldrack and Packard, 2003; Poldrack and Rodriguez, 2003), including explicit motor-sequence learning (Schendan et al., 2003), leads to recruitment of the hippocampal formation. Our results are consistent with this notion, and we speculate that as the output capabilities of the system increase following sleep, the hippocampal need for ordering these individual motor elements (key-presses) in a correct temporal series must concomitantly increase. Secondly, activation patterns within the prefrontal cortex showed a dissociation 
following sleep, with activity increasing in the right anterior medial prefrontal cortex, but decreasing in the left fronto-polar region. These differences are in accord with the sequencing model put forward by Koechlin et al. (1999, 2000, 2002), proposing that while fronto-polar regions are critical for the online conscious search of sequential patterns and inference, the anterior medial prefrontal regions are utilized for the planning and building of motor sequences. Advancing this model, our results would suggest that overnight motor learning is accompanied by enhanced sequence planning in the anterior medial prefrontal cortex, which in addition to the increased striate activity (Doyon et al., 2003), facilitates the construction of more efficient motor-sequence patterns required for optimizing within-sequence transitions (Kuriyama et al., 2004). In contrast, due to the overnight sleep-dependent learning benefit (Walker et al., 2002, 2003a,b; Fischer et al., 2002; Korman et al., 2003) and greater task automaticity (Kuriyama et al., 2004), the need for online monitoring of skill performance decreases, resulting in reduced fronto-polar activation.

Additional regions of decreased signal intensity were found throughout the partial cortex following sleep, a pertinent finding for several reasons. Firstly, decreased activity in the parietal lobes has been consistently reported in relation to enhanced motor skill learning (Seitz et al., 1990; Toni et al., 1998; Muller et al., 2002), a phenomena believe to reflect improved sequence automation. Secondly, Huber et al. (2004) recently reported that daytime learning of a motor adaptation task results in a discrete increase in the subsequent amount of NREM slow-wave activity over the parietal cortex, and that this slow-wave increase was proportional to the amount of delayed learning that developed the next day; signifying a potential link between overnight plastic changes in the parietal cortex and the degree of sleep-dependent improvement. Supporting these findings, and based on our current results, we hypothesize that as learning and task optimization is improved overnight (Kuriyama et al., 2004), there is a corresponding decreased need for conscious mapping of spatial relationships between finger movements and position (Seitz et al., 1990; Toni et al., 1998; Muller et al., 2002), the consequence of which is reduced post-sleep parietal involvement.

Finally, there were also regions of decreased activation in the left limbic cortical network of the insula and temporal pole (Augustine, 1996) following sleep. While remaining speculative, these post-sleep decreases may reflect greater ease of task performance due to the overnight learning benefit and improved skill proficiency (Walker et al., 2002), thereby reducing the emotional burden and hence limbic involvement (Brooks, 1986; Seitz et al., 1990). Indeed, a recent study investigating functional brain changes associated with sleep-dependent learning of a visual skill task similarly report decreased post-sleep activation in the temporal pole (Walker et al., in press), and may represent a common underlying feature of sleepdependent plasticity across both procedural sensory and motor skill memory domains.

\section{CONCLUSIONS}

In summary, these findings describe a systems-level change in the neural representation of a learned motorsequence following a night of sleep. This pattern of overnight plasticity, associated with sleep-dependent memory processing, holds important implications for the learning of countless real-life motor skills, and also suggests its potential impairment or complete absence in psychiatric disorders expressing a failure of normal sleep-dependent motor learning (Manoach et al., 2004). It also offers the exciting prospect that sleep plays a significant role in clinical rehabilitation and recovery of movement function following insults to the motor system, such as stroke.

Acknowledgments-The authors wish to thank Drs. Christian Gaiser, Seung-Schik Yoo, Edward Pace-Schott and Edwin Robertson for their guidance and helpful comments regarding these findings, and Alex Morgan for technical assistance. This work was supported in part by grants from the National Institutes of Health (MH 48,832; MH 65,292; MH 67,754; MH 69,935 and NS 45,049) and the Dana Foundation.

\section{REFERENCES}

Atienza M, Cantero JL, Stickgold R (2004) Posttraining sleep enhances automaticity in perceptual discrimination. $\mathrm{J}$ Cogn Neurosci 16:53-64.

Augustine JR (1996) Circuitry and functional aspects of the insular lobe in primates including humans. Brain Res Brain Res Rev 22:229-244.

Benington JH, Frank MG (2003) Cellular and molecular connections between sleep and synaptic plasticity. Prog Neurobiol 69:71-101.

Brooks VB (1986) How does the limbic system assist motor learning? A limbic comparator hypothesis. Brain Behav Evol 29:29-53.

Doyon J, Penhune V, Ungerleider LG (2003) Distinct contribution of the cortico-striatal and cortico-cerebellar systems to motor skill learning. Neuropsychologia 41:252-262.

Fenn KM, Nusbaum HC, Margoliash D (2003) Consolidation during sleep of perceptual learning of spoken language. Nature 425: 614-616.

Fischer S, Hallschmid M, Elsner AL, Born J (2002) Sleep forms memory for finger skills. Proc Natl Acad Sci U S A 99:11987-11991.

Friston K, Holmes A, Worsley K, Poline J, Frith C, Frackowiak R (1995) Statistical parametric maps in functional imaging: a general linear approach. Hum Brain Mapp 2:189-210.

Gaab N, Paetzold M, Becker M, Walker MP, Schlaug G (2004) The influence of sleep on auditory learning: A behavioral study. Neuroreport 15:731-734.

Gais S, Plihal W, Wagner U, Born J (2000) Early sleep triggers memory for early visual discrimination skills. Nat Neurosci 3:13351339.

Graves L, Pack A, Abel T (2001) Sleep and memory: a molecular perspective. Trends Neurosci 24:237-243.

Huber R, Ghilardi MF, Massimini M, Tononi G (2004) Local sleep and learning. Nature 430:78-81.

Karni A, Meyer G, Jezzard P, Adams MM, Turner R, Ungerleider LG (1995) Functional MRI evidence for adult motor cortex plasticity during motor skill learning. Nature 377:155-158.

Karni A, Tanne D, Rubenstein BS, Askenasy JJ, Sagi D (1994) Dependence on REM sleep of overnight improvement of a perceptual skill. Science 265:679-682.

Koechlin E, Basso G, Pietrini P, Panzer S, Grafman J (1999) The role of the anterior prefrontal cortex in human cognition. Nature 399:148-151. 
Koechlin E, Corrado G, Pietrini P, Grafman J (2000) Dissociating the role of the medial and lateral anterior prefrontal cortex in human planning. Proc Natl Acad Sci U S A 97:7651-7656.

Koechlin E, Danek A, Burnod Y, Grafman J (2002) Medial prefrontal and subcortical mechanisms underlying the acquisition of motor and cognitive action sequences in humans. Neuron 35:371-381.

Korman M, Raz N, Flash T, Karni A (2003) Multiple shifts in the representation of a motor sequence during the acquisition of skilled performance. Proc Natl Acad Sci U S A 100:12492-12497.

Kuriyama K, Stickgold R, Walker MP (2004) Sleep-dependent learning and motor skill complexity. Learn Mem 11:705-713.

Manoach DS, Caine MS, Vangel MG, Kurana A, Goff DC, Stickgold R (2004) A failure of sleep-dependent procedural learning in chronic, medicated schizophrenia. Biol Psychiatry 56:951-956.

Muller RA, Kleinhans N, Pierce K, Kemmotsu N, Courchesne E (2002) Functional MRI of motor sequence acquisition: effects of learning stage and performance. Brain Res Cogn Brain Res 14:277-293.

Ohyama T, Nores WL, Murphy M, Mauk MD (2003) What the cerebellum computes. Trends Neurosci 26:222-227.

Pascual-Leone A, Nguyet D, Cohen LG, Brasil-Neto JP, Cammarota A, Hallett M (1995) Modulation of muscle responses evoked by transcranial magnetic stimulation during the acquisition of new fine motor skills. J Neurophysiol 74:1037-1045.

Penhune VB, Doyon J (2002) Dynamic cortical and subcortical networks in learning and delayed recall of timed motor sequences. J Neurosci 22:1397-1406.

Poldrack RA, Packard MG (2003) Competition among multiple memory systems: converging evidence from animal and human brain studies. Neuropsychologia 41:245-251.

Poldrack RA, Rodriguez P (2003) Sequence learning: what's the hippocampus to do? Neuron 37:891-893

Robertson EM, Pascual-Leone A, Press DZ (2004) Awareness modifies the skill-learning benefits of sleep. Curr Biol 14:208-212.

Sakai K, Ramnani N, Passingham RE (2002) Learning of sequences of finger movements and timing: frontal lobe and action-oriented representation. J Neurophysiol 88:2035-2046.

Schendan HE, Searl MM, Melrose RJ, Stern CE (2003) An FMRI study of the role of the medial temporal lobe in implicit and explicit sequence learning. Neuron 37:1013-1025.

Schmahmann JD, Doyon J, McDonald D, Holmes C, Lavoie K, Hurwitz AS, Kabani N, Toga A, Evans A, Petrides M (1999) Three-dimen- sional MRI atlas of the human cerebellum in proportional stereotaxic space. Neuroimage 10:233-260.

Seitz RJ, Roland E, Bohm C, Greitz T, Stone-Elander S (1990) Motor learning in man: a positron emission tomographic study. Neuroreport 1:57-60.

Smith C, MacNeill C (1994) Impaired motor memory for a pursuit rotor task following stage 2 sleep loss in college students. J Sleep Res 3:206-213.

Stickgold R, James L, Hobson JA (2000a) Visual discrimination learning requires sleep after training. Nat Neurosci 3:1237-1238.

Stickgold R, Whidbee D, Schirmer B, Patel V, Hobson JA (2000b) Visual discrimination task improvement: A multi-step process occurring during sleep. J Cogn Neurosci 12:246-254.

Toni I, Krams M, Turner R, Passingham RE (1998) The time course of changes during motor sequence learning: a whole-brain fMRI study. Neuroimage 8:50-61.

Tononi G, Cirelli C (2001) Some considerations on sleep and neural plasticity. Arch Ital Biol 139:221-241.

Ungerleider LG, Doyon J, Karni A (2002) Imaging brain plasticity during motor skill learning. Neurobiol Learn Mem 78:553-564.

Walker MP (2005) A refined model of sleep and the time course of memory formation. Behav Brain Sci, in press.

Walker MP, Brakefield T, Allan Hobson J, Stickgold R (2003a) Dissociable stages of human memory consolidation and reconsolidation. Nature 425:616-620.

Walker MP, Brakefield T, Morgan A, Hobson JA, Stickgold R (2002) Practice with sleep makes perfect: sleep dependent motor skill learning. Neuron 35:205-211.

Walker MP, Brakefield T, Seidman J, Morgan A, Hobson JA, Stickgold $\mathrm{R}$ (2003b) Sleep and the time course of motor skill learning. Learn Mem 10:275-284.

Walker MP, Stickgold R (2004) Sleep-dependent learning and memory consolidation. Neuron 44:121-133.

Walker MP, Stickgold R, Jolesz FA, Yoo SS (2005) The functional anatomy of sleep-dependent visual skill learning. Cereb Cortex, in press.

Worsley KJ, Marrett S, Neelin P, Vandal AC, Friston KJ, Evans AC (1996) A unified statistical approach for determining significant voxels in images of cerebral activation. Hum Brain Mapp $4: 58-73$. 